\title{
Lapidus arthrodesis in combination with arthrodesis of the first metatarsophalangeal joint-biomechanical cadaver study comparing two methods of fixation
}

\author{
Radek Kunovsky ${ }^{\mathrm{a}}$, Jan Kocis ${ }^{\mathrm{b}}$, Tomas Navratc , Petr Vosynekc, Tomas Pink ${ }^{\mathrm{a}, \mathrm{b}}$, Marek Joukal ${ }^{\mathrm{d}}$, Igor Cizmar
}

\begin{abstract}
Aims. To assess the results of a biomechanical test of cadaveric specimens, comparing 2 methods of fixation of modified Lapidus arthrodesis in combination with arthrodesis of the first metatarsophalangeal joint.

Methods. A total of 12 cadaveric specimens were used in the test. Arthrodesis of the first MTP joint was in all patients fixed with a Variable Angle LCP 1st MTP Fusion Plate 2.4/2.7. Two methods of fixation of the Lapidus arthrodesis were compared, i.e. fixation with two screws in the PS (plate-screw) version versus fixation with X-Locking Plate 2.4/2.7 in the PP (plate-plate) version. Measurements were obtained with the use of a testing machine ZWICK Z 020-TND with an optical device Mercury RT for measuring deformities. Each specimen was subjected to 3 loading options, a. displacement $5 \mathrm{~mm}$, the support is placed under the proximal phalanx, b. displacement $5 \mathrm{~mm}$, the support is placed under the first metatarsal head and $c$. load to failure, the support is placed under the first metatarsal head.

Results. In all specimens the PS construct showed a statistically considerably higher stiffness than the PP construct. In all specimens treated with the PP construct the load to failure was lower than in the PS construct. For loading mode a., at a significance level of $0.05(P<0.05)$, the $P$-value was 0.036 , for mode b. the $P$-value was 0.007 and for loading mode c. the $P$-value was 0.006 . In addition, age-related decrease in stiffness of the specimen was proved at a significance level of $5 \%(P=0.004)$.
\end{abstract}

Conclusion. In all the three loading modes, the PS (plate-screw) construct showed a statistically higher stiffness than the PP (plate-plate) construct.

Key words: arthrodesis, metatarsophalangeal joint, Lapidus, cadaver, biomechanical study

Received: February 5, 2021; Revised: April 3, 2021; Accepted: April 19, 2021; Available online: May 11, 2021 https://doi.org/10.5507/bp.2021.025

(0) 2022 The Authors; https://creativecommons.org/licenses/by/4.0/

${ }^{a}$ Department of Clinical Orthopedics, Trauma Hospital, Brno, Czech Republic

${ }^{b}$ Department of Trauma Surgery, Faculty of Medicine, Masaryk University, Brno, Czech Republic

Institute of Solid Mechanics, Mechatronics and Biomechanics, Faculty of Mechanical Engineering, Technical University, Brno, Czech Republic dInstitute of Anatomy, Faculty of Medicine, Masaryk University, Brno, Czech Republic

eEducational Center for Practical Anatomy, Brno, Czech Republic

Corresponding author: Radek Kunovsky, e-mail: radek.kunovky@seznam.cz

\section{INTRODUCTION}

There are several types of procedures to treat severe hallux valgus. If the angle between first and second metatarsal (MTT) is greater than 20 degrees, proximal osteotomy (open wedge, close wedge or crescentic osteotomy) of the first MTT are preferable. Proximal osteotomy of the first MTT salvages the joint between the first MTT and the medial cuneiform. In some cases, after the proximal osteotomy has healed, this joint is overloaded. The first MTT turns inward (varus), which results in loss of the correction and recurrence of the deformity. For these reasons, salvage proximal osteotomy of the first MTT is only performed in younger patients with a completely stable first metatarsal-cuneiform joint without osteoarthritic changes. We prefer modified Lapidus arthrodesis between the base of the first MTT and the medial cuneiform for the correction of severe hallux valgus. The modified Lapidus surgical procedure is combined with a procedure on the first metatarsophalangeal joint. In case of a reducible defect, we perform a salvaging procedure. Soft-tissue techniques are often combined with an Akin osteotomy of the proximal phalanx. We use an LCP plate and lag screws for fixation of the arthrodesis of the first metatarsophalangeal joint and 2 BOLD screws or LCP X plate in combination with a lag screw for fixation of the Lapidus arthrodesis.

In case of a marked valgus deformity with osteoarthritis of the first metatarsophalangeal (MTP) joint and varus deformity of the first metatarsal (MTT), we choose a twolevel arthrodesis to correct the axis of the entire first ray of the foot, an excessive hallux valgus angle as well as the 1-2 intermetatarsal angle. This usually concerns patients with rheumatoid arthritis. This procedure also minimizes the risk of recurrence of the deformity. A combined twolevel arthrodesis increases rigidity of the entire first ray of the foot. Arthrodesis of the first tarsometatarsal (TMT) joint is exposed to a higher load when a stable arthrodesis of the first MTP joint has been performed as compared to cases without arthrodesis of that joint. In these cases, 
Lapidus arthrodesis should be as stable as possible in order to minimize the risk of nonunion.

Although the outcomes of Lapidus arthrodesis are very good, nonunion or malunion occur in $5 \%-15 \%$ of cases ${ }^{1}$. Myerson et al. report incidence of nonunions at $4.5 \%$ and Sangeorzan and Hansen at up to $13 \%$ (ref. $^{2,3}$ ). Roukis in his meta-analysis reports nonunion incidence after arthrodesis of the first MTP joint in 5.4\% (153 of 2818 ) and malunion in $6.1 \%$ of patients ${ }^{4}$. Mainly for this reason there appeared a number of experimental biomechanical studies comparing stability of fixation of arthrodesis of the first MTP joint as well as stability of arthrodesis between the first metatarsal (MTT) and the medial cuneiform. Arthrodesis can be performed with the use of various fixation devices, including the simplest Kirschner wires, various types of screws, memory staples or LCP systems with an interfragmentary screw.

Biomechanical studies show that the most stable option is fixation using an LCP plate in combination with a lag screw. Our work is experimental. For this reason, we intentionally chose not to use a lag screw for fixation, although we standardly use this in clinical practice. The result of the experiment using a lag screw would be clear-cut, its impact on increasing strength (stability) has already been proven by other authors ${ }^{5}$. In 3 of 31 patients with rheumatoid arthritis, Popelka et al. combined a Lapidus arthrodesis with an arthrodesis of the first metatarsophalangeal joint ${ }^{6}$. In his series of 18 feet with severe hallux valgus and an increased IMA 1-2, Rippstein treated patients using MTP-1 fusion with additional proximal correction (Mau osteotomy or a modified Lapidus procedure). All patients were satisfied or very satisfied with the results; there were no intraoperative complications. The IMA 1-2 significantly improved from a mean of 18.8 degrees before surgery to 4.6 degrees on follow up $(P<0.001)$ and the HVA decreased from a mean of 49.9 degrees to 9.7 degrees $(P<0.001)$, respectively ${ }^{7}$. However, we have not found any biomechanical study in the available literature with the combined two-level arthrodesis presented in our study.

\section{MATERIALS AND METHODS}

\section{Hypothesis of the biomechanical study}

Fixation by 2 BOLD screws will be more solid than fixation by LCP X plate at combination arthrodesis of the first MTP joint with arthrodesis of the first TMT joint.

All cadaveric specimens were treated with arthrodesis of the first MTP joint in combination with arthrodesis of the first TMT joint according to modified Lapidus.

Arthrodesis of the first MTP joint was in all patients performed with Variable Angle LCP $1^{\text {st }}$ MTP Fusion Plate 2.4/2.7 (Synthes, Switzerland) fixed by 3 screws 2.7 to the proximal phalanx and 3 screws 2.7 to the first MTT. In case of PS (plate-screw) construct, we performed modified Lapidus arthrodesis with the use of 2 screws (BOLD $\mathbb{R}$ Compression Screw, Integra, USA) (Fig. 1). The BOLD screw is a titanium alloy compression $3.0 \mathrm{~mm}$ screw, cannulated, double helix thread design for increased bone

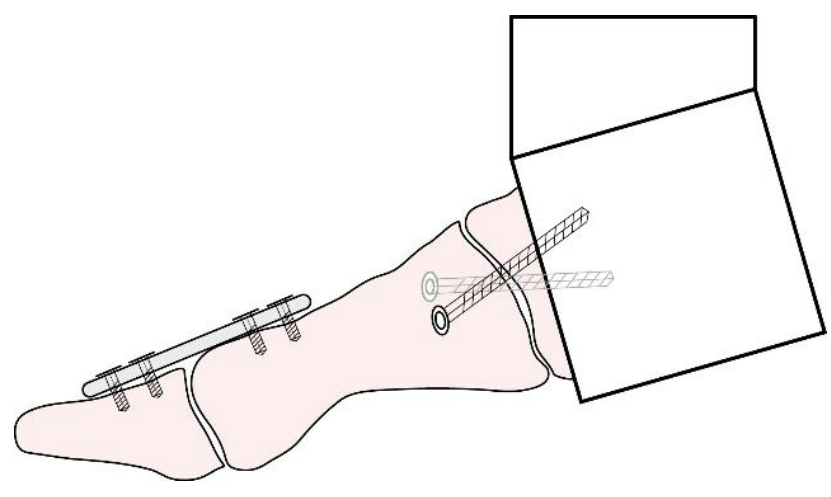

Fig. 1. PS (plate-screw) construct.

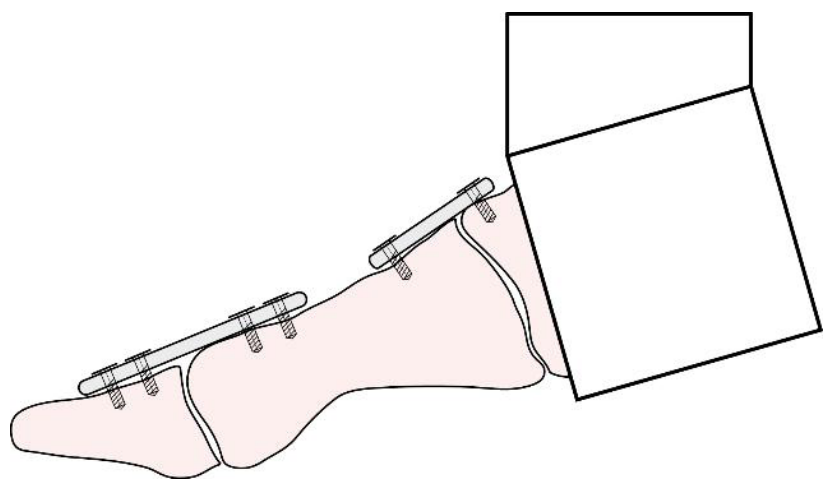

Fig. 2. PP (plate-plate) construct.

purchase, in order to optimize compression, the head has to be totally embedded. We used 2 BOLD screws.

In case of PP (plate-plate) construct, we implanted X-Locking Plate 2.4/2.7 (Synthes, Switzerland) fixed by 2 screws 2.7 to the medial cuneiform and 2 screws 2.7 to the first MTT base (Fig. 2).

Testing included a total of 12 cadaver feet ( 6 pairs). All specimens were obtained from women with a mean age at death of 76 years (range, 55-87). Comparison was performed on the right and left cadaver feet always of the same donor in order to ensure equal anatomical conditions of the test (bone length, width and density). The specimens included the proximal phalanx, the first MTT, the medial cuneiform, the intermediate cuneiform, the second MTT base and the navicular. Prior to testing, specimens were defrosted from $-20{ }^{\circ} \mathrm{C}$ to room temperature.

\section{Operative technique - arthrodesis of the first MTP joint}

The cadaveric specimen was cleared of all soft tissues; joint capsule and collateral ligaments of the first MTP joint were removed, while articular cartilage was left in place. We prefer arthrodesis in 10-15 degree of valgus, neutral rotation and 10-15 degrees of dorsiflexion. Selftapping $2.7 \mathrm{~mm}$ LCP screws are gradually predrilled. They can be inserted at a variable angle of 15 degrees. Three screws are driven into the first MTT and another 3 screws into the proximal phalanx. The screws need not penetrate the second cortex. 
Operative technique - PS (plate-screw) construct, modified Lapidus arthrodesis fixed with 2 BOLD screws

After the necessary preparation, arthrodesis is fixed with 2 BOLD screws (length $30 \mathrm{~mm}$ ), one inserted from the base of the first to the base of the second MTTs and another from the base of the first MTT obliquely to the medial cuneiform. Position of plates and screws in all specimens is checked by X-ray (Fig. 1).

Operative technique - PP (plate-plate) construct, modified Lapidus arthrodesis fixed with X-locking plate

Unlike in vivo operations, articular cartilage between the first MTT base and the medial cuneiform is left in place. Following temporary fixation with a Kirschner wire, X-Locking Plate is placed dorsally and fixed with 2 screws (length $24 \mathrm{~mm}$ ) inserted into the first MTT base and 2 screws (length $28 \mathrm{~mm}$ ) inserted into the medial cuneiform (Fig. 2).

\section{Preparation of specimens and mounting in the testing machine}

A special preparation was developed to mount specimens in the testing machine, which allowed specimens to be swapped quickly during the experiment. Specimens were fixed in the same position with Duracryl (methyl methacrylate resin). All specimens were mounted in the testing machine (Fig. 3).

Measurements were obtained with the use of a universal testing machine ZWICK Z 020-TND (Zwick Roell, Ulm, Germany) (Fig. 4), a mechanical, computer aided device for compression and tensile load tests, with the applied loads of up to $20 \mathrm{kN}$. The machine is equipped with Multisens extensometer with $0.1 \mu \mathrm{m}$ measuring accuracy. The software allows setup of various load cycles. Part of the system is an optical device Mercury RT (real-time tracking system) for measuring deformities. The system comprises 2 cameras, light and control software (Fig. 5).

\section{Load testing process}

The specimen under load was deviated from the horizontal by about $=15^{\circ}$. The specimen was subjected to a constant downward force, as a result, it was exposed to a combination of bending and compression. In the analysis of the results, the terms stiffness and compliance of an object are used. During mechanical strain applied to an object, stiffness is understood as the magnitude of its deformation in response to application of a force. The opposite value is called compliance.

During testing, time dependent variation of the force was recorded on the testing machine and time dependent magnitude of deformation on the optical device. The combination of data from the two systems produced force-to-displacement relationship $(\mathrm{F}-\mathrm{w})$. In order to eliminate the stiffness-to-specimen length (L) relationship, F - w was converted to $\mathrm{FL}^{3}$ - w relationship. An example of such dependence for specimen number 6 is shown in Fig 6.

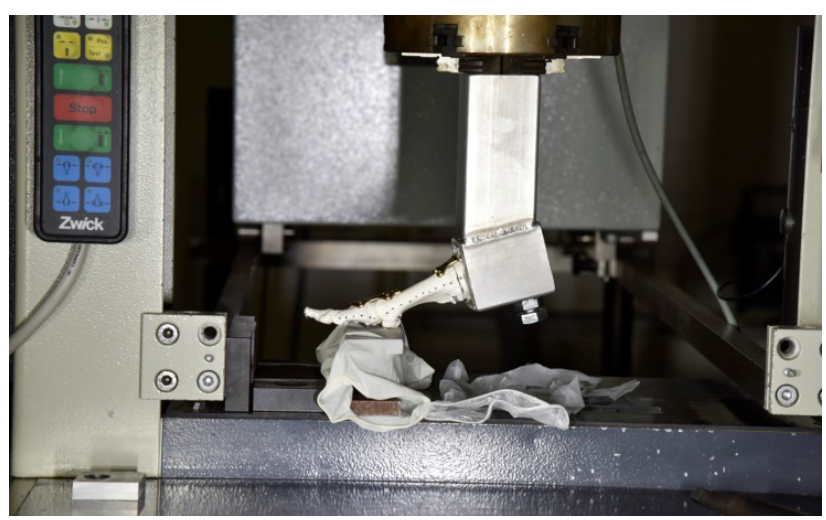

Fig. 3. Specimen mounted in the testing machine.

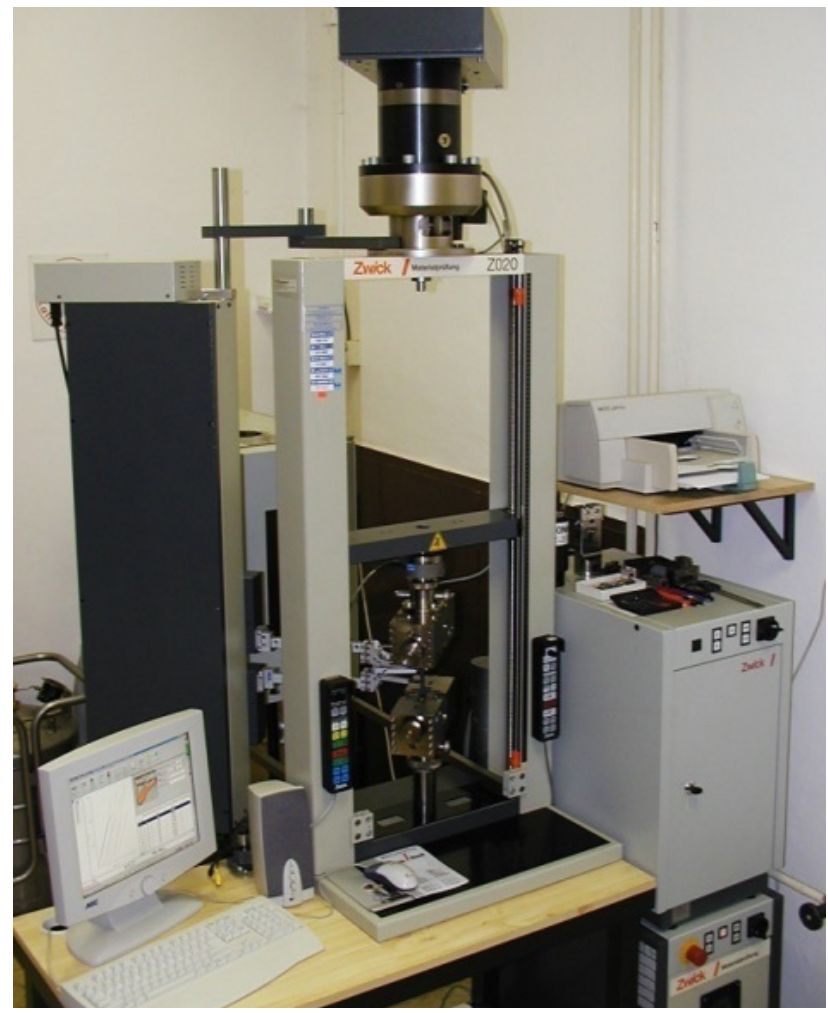

Fig. 4. ZWICK Z 020-TND testing machine.

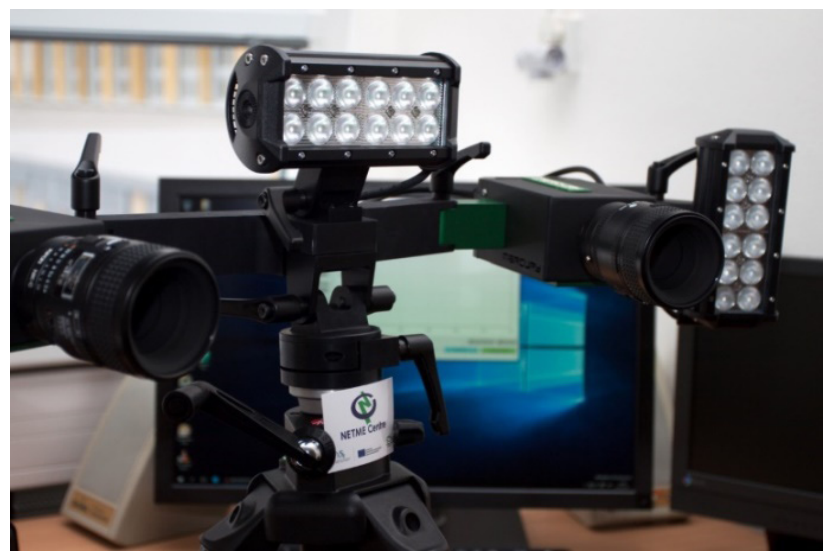

Fig. 5. Mercury RT optical device for measuring deformities. 

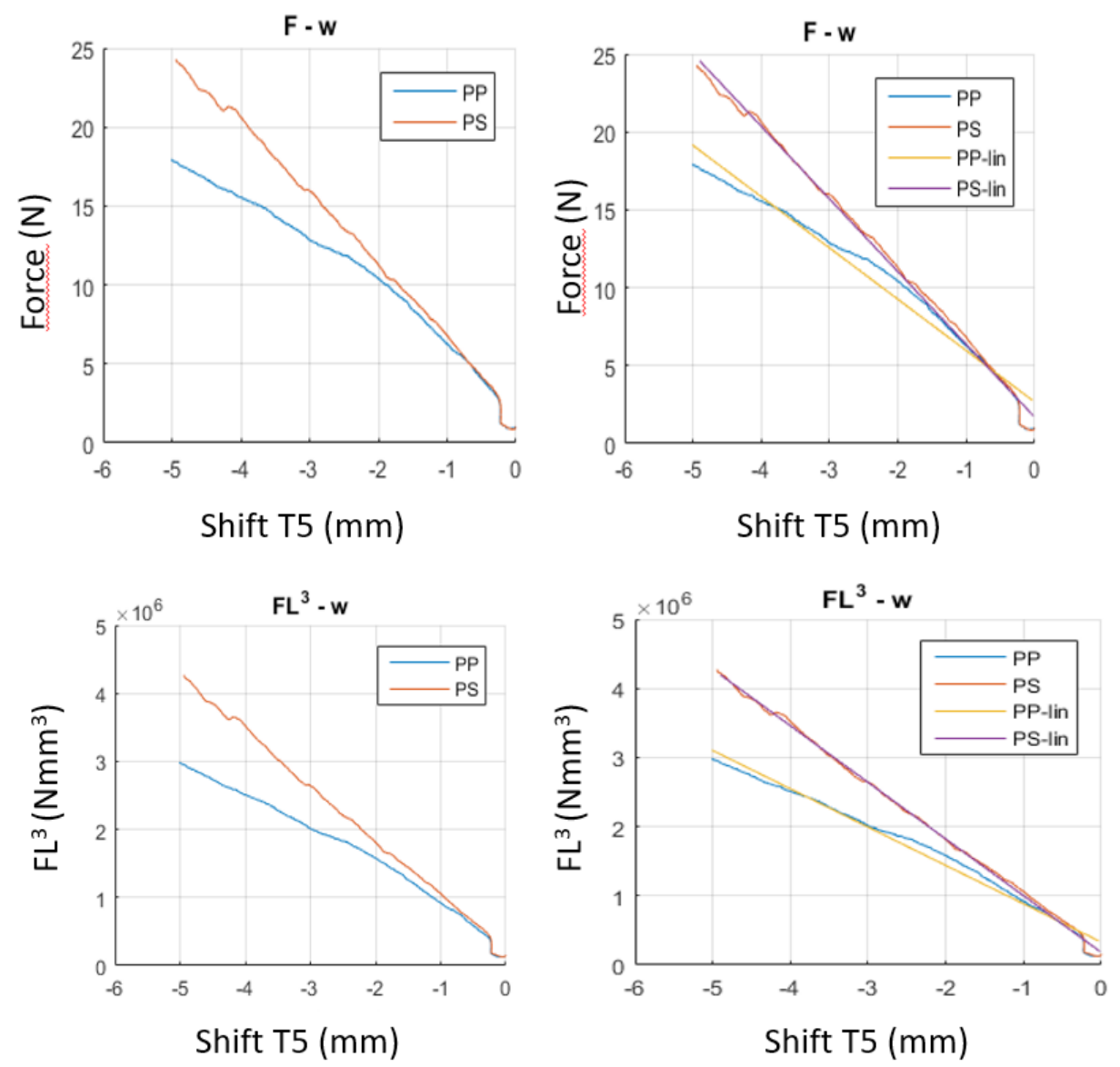

Fig. 6. Results for specimen 6, loading b. PP (plate-plate), PS (plate-screw), PP-lin (linear regression), PS-lin (linear regression)
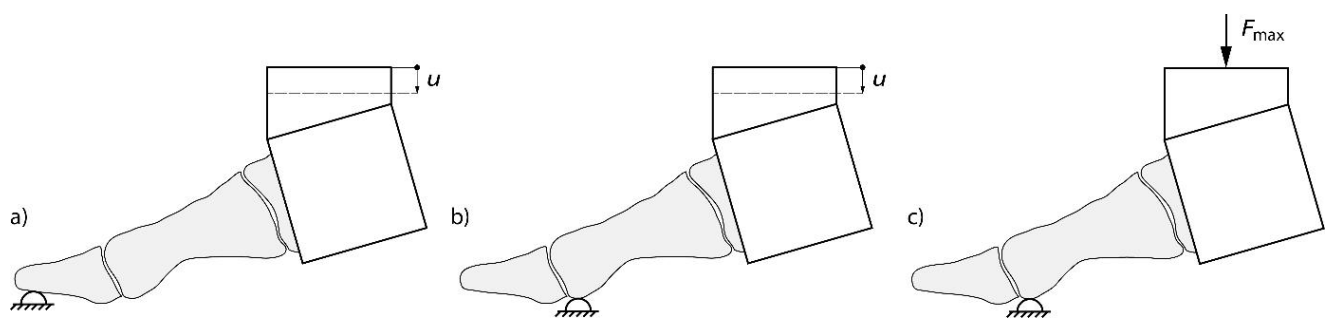

Fig. 7. Configuration of specimens under load.

\section{Loading options}

Each specimen was subjected to the following 3 loading options:

a) displacement $\mathrm{u}=5 \mathrm{~mm}$, the support is placed under the proximal phalanx, load force needed for compression by $5 \mathrm{~mm}$ is measured (Fig. 7a)

b) displacement $\mathrm{u}=5 \mathrm{~mm}$, the support is placed under the first MTT head, load force needed for compression by $5 \mathrm{~mm}$ is measured (Fig. 7b)

c) load to failure, the support is placed under the first MTT head (Fig. 7c)

\section{RESULTS}

\section{Results for loading a}

The two methods of fixation were compared using the t-test. We tested whether the mean difference value is zero versus the alternative hypothesis that it is not. We calculated a $P$-value of 0.036 , so at a significance level of $0.05(P<0.05)$, we rejected the zero-value hypothesis of the mean value. This result can be interpreted in such a way that PS fixation has a statistically significantly greater stiffness than PP fixation.

\section{Results for loading $b$}

The mean and median values of bending stiffness are higher for PS fixation. On calculation in the t-test, we obtain a $P$-value of 0.007 , so at a significance level of 
Table 1. Bending stiffness values $\left[\mathrm{MPa} \cdot \mathrm{mm}^{4}\right]$ for loading a.

\begin{tabular}{lrrrrrrr}
\hline Specimen & 1 & 2 & 3 & 4 & 5 & 6 & Model \\
\hline PS & 1178623 & 344880 & 1480114 & 1336540 & 842165 & 946050 & 1239870 \\
PP & 1046633 & 323546 & 710366 & 705624 & 654012 & 626761 & 1235482 \\
Difference PS-PP & 131990 & 21334 & 769748 & 630916 & 188153 & 319290 & 4388 \\
\hline
\end{tabular}

Table 2. Descriptive statistical data for bending stiffness in loading option a.

\begin{tabular}{lccrrrc}
\hline & $\begin{array}{c}\text { Number of } \\
\text { specimens }\end{array}$ & $\begin{array}{c}\text { Arithmetic } \\
\text { mean }\end{array}$ & $\begin{array}{c}\text { Standard } \\
\text { deviation }\end{array}$ & Median & Minimum & Maximum \\
\hline PS & 6 & 1021395 & 407415 & 1062337 & 344880 & 1480114 \\
PP & 6 & 677824 & 230885 & 679818 & 323546 & 1046633 \\
\hline
\end{tabular}

Table 3. Bending stiffness values $\left[\mathrm{MPa} \cdot \mathrm{mm}^{4}\right]$ for loading option $\mathrm{b}$.

\begin{tabular}{lrrrrrrr}
\hline Specimen & 1 & 2 & 3 & 4 & 5 & 6 & Model \\
\hline PS & 1032608 & 256782 & 709320 & 622508 & 465867 & 855510 & 1436981 \\
PP & 836843 & 229081 & 534748 & 559021 & 303054 & 620240 & 1320456 \\
Difference PS-PP & 195765 & 27701 & 174573 & 63487 & 162814 & 235269 & 116525 \\
\hline
\end{tabular}

Table 4. Descriptive statistical data for bending stiffness in loading option b.

\begin{tabular}{lcccccr}
\hline & $\begin{array}{c}\text { Number of } \\
\text { specimens }\end{array}$ & $\begin{array}{c}\text { Arithmetic } \\
\text { mean }\end{array}$ & $\begin{array}{c}\text { Standard } \\
\text { deviation }\end{array}$ & Median & Minimum & Maximum \\
\hline PS & 6 & 657099 & 276087 & 665914 & 256782 & 1032608 \\
PP & 6 & 513831 & 220748 & 546885 & 220748 & 836843 \\
\hline
\end{tabular}

Table 5. Maximum force values [N] for loading option c.

\begin{tabular}{rrrrrrrr}
\hline Specimen & 1 & 2 & 3 & 4 & 5 & 6 & model \\
\hline PS & 80 & 38 & 54 & 64 & 53 & 75 & 94 \\
PP & 70 & 30 & 30 & 28 & 20 & 34 & 89 \\
Difference PS - PP & 10 & 8 & 24 & 36 & 33 & 41 & 5 \\
\hline
\end{tabular}

Table 6. Descriptive statistical data for bending stiffness in loading option c.

\begin{tabular}{lcccccc}
\hline & $\begin{array}{c}\text { Number of } \\
\text { specimens }\end{array}$ & $\begin{array}{c}\text { Arithmetic } \\
\text { mean }\end{array}$ & $\begin{array}{c}\text { Standard } \\
\text { deviation }\end{array}$ & Median & Minimum & Maximum \\
\hline PS & 6 & 60.7 & 15.5 & 59.0 & 38.0 & 80.0 \\
PP & 6 & 35.3 & 17.6 & 30.0 & 20.0 & 70.0 \\
\hline
\end{tabular}

$0.05(P<0.05)$, we rejected the zero-value hypothesis of the mean value, PS fixation has statistically significantly greater stiffness than PP fixation.

\section{Results for loading c}

The aim of option c was to determine the maximum force leading to failure of fixation between the first MTT and the medial cuneiform. In all specimens, the screws in the bone were loosened at the maximum load. Screws were loosened five times from first metatarsal bone and one time from medial cuneiform bone. Maximum force values for individual specimens are summarized in Table 5. An example of the effect of load force variation on specimen deformation is shown for specimen 6 in Fig. 8 . The results for the model specimen were excluded from further analysis. Descriptive statistical data for maximum forces is summarized in Table 6. Graphical interpretation of maximum forces is presented in the boxplot in Fig. 9.

The two methods of fixation were compared. On calculation in the t-test, we obtain a $P$-value of 0.006 , so at a significance level of $0.05(P<0.05)$, we rejected the zero-value hypothesis of the mean value, which showed a statistically significantly higher load to failure for PS fixation than for PP construct. This result was also observed in the model sample. Fig. 10, 11 show gapping of the fixed joint with a load force of $30 \mathrm{~N}$, namely $12 \mathrm{~mm}$ with the PP construct and $2 \mathrm{~mm}$ with the PS construct.

The results of all types of loading, loading a, b and c, clearly show significantly higher fixation stiffness of the arthrodesis of the first tarsometatarsal joint with 2 BOLD screws compared to fixation with an X plate.

The result of this study confirmed our hypothesis. 


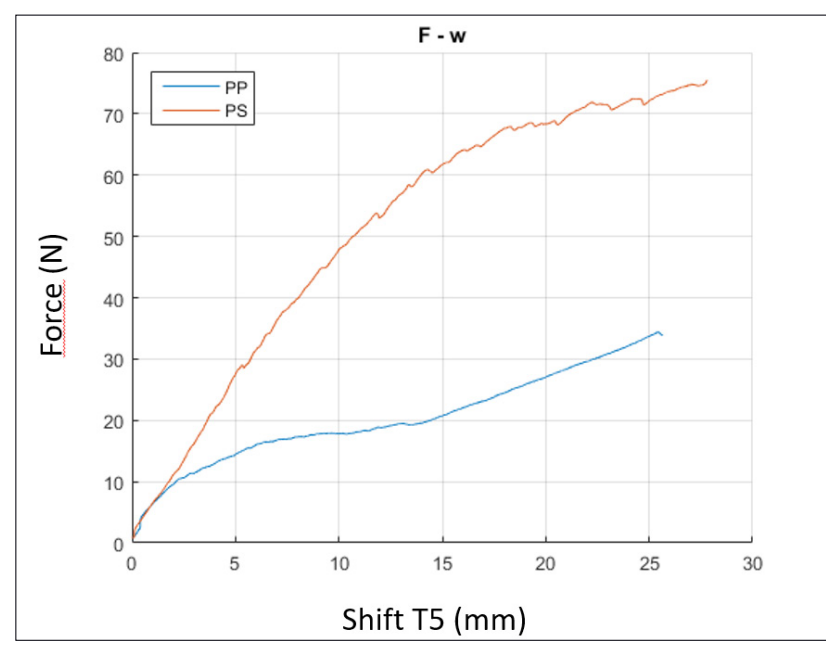

Fig. 8. Results for specimen 6 loading c.

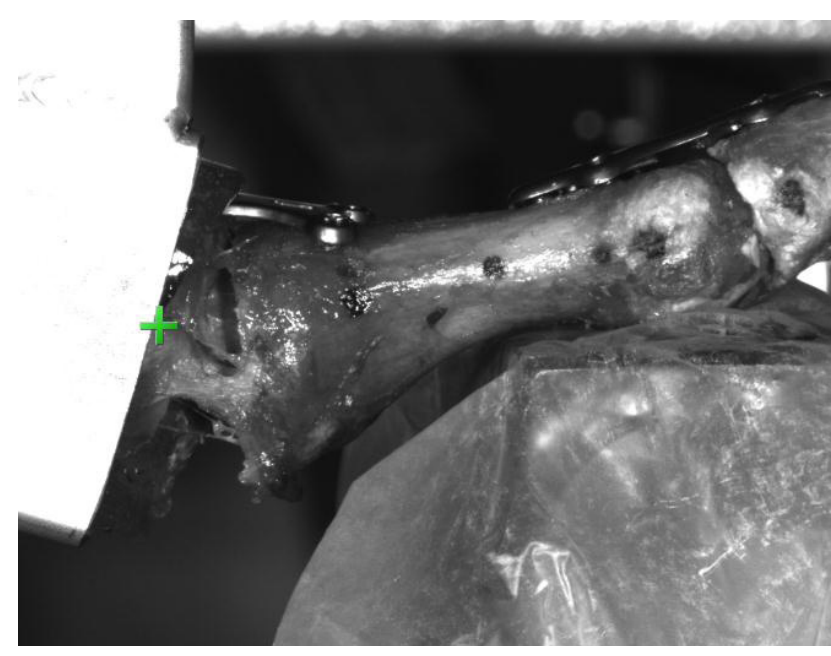

Fig. 10. Joint gapping in specimen 6 after application of $30 \mathrm{~N}$ force, PP fixation.

\section{Effect of age of specimen on its stiffness}

Correlation of measurements of stiffness and age of specimens reveals a relationship between these values. Age-related decrease in stiffness of specimens is given by degradation of bone material properties. Due to a small number of specimens, linear regression function was chosen for decrease of stiffness, with the following relationship identified: stiffness $=1548$ 005-12555.age. Regression function was statistically significant, at the level of $5 \%(P=0.004)$.

\section{DISCUSSION}

There are several types of procedures to treat severe hallux valgus. Where the angle between the first and second MTT exceeds 20 degrees, proximal osteotomy of the first MTT should be used. After healing of proximal osteotomy, the first TMT joint may get overloaded, turning the first MTT to varus, which results in loss of the correction and recurrence of the deformity. We use salvage

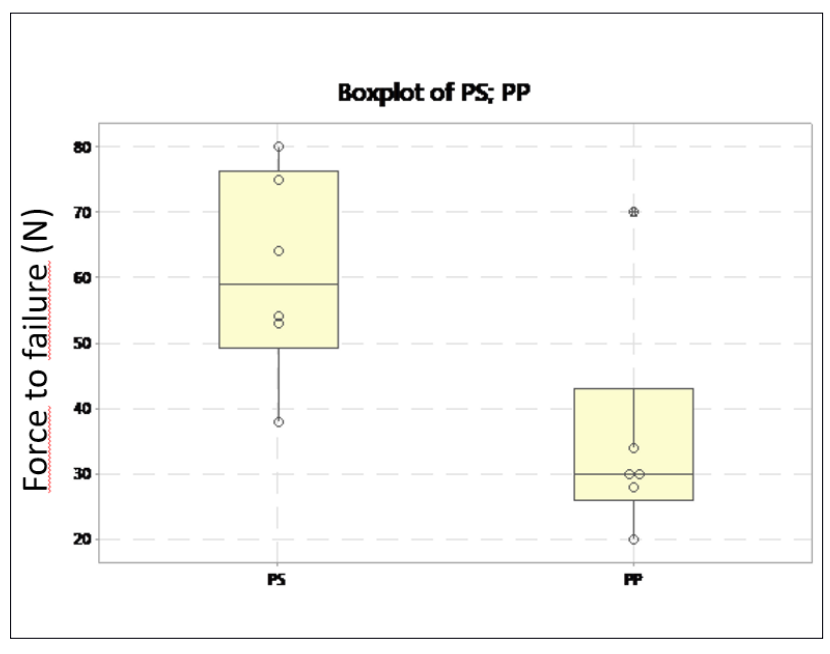

Fig. 9. Force to failure of fixation loading c.

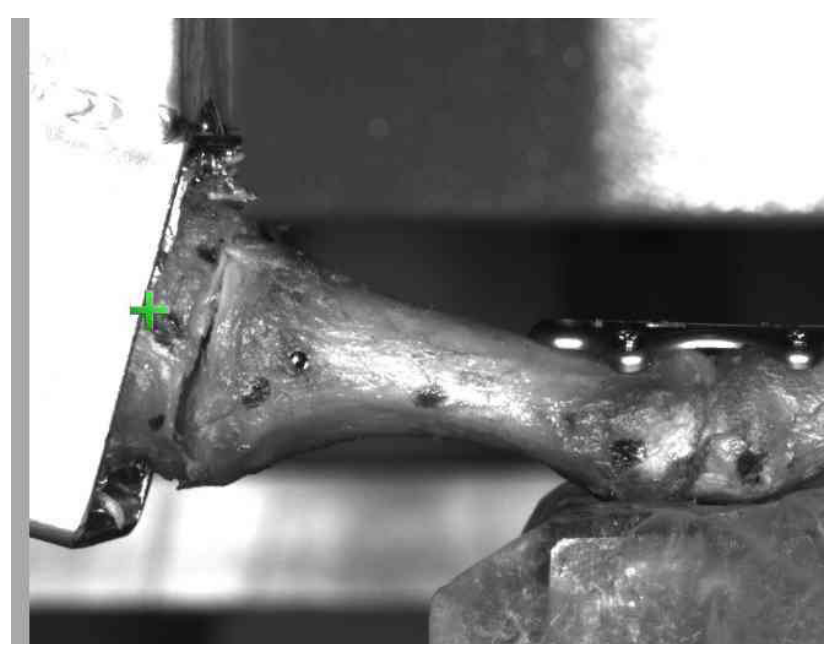

Fig. 11. Joint gapping in specimen 6 after application of $30 \mathrm{~N}$ force, PS fixation.

proximal osteotomy of the first MTT only in patients with a fully stable first metatarsal cuneiform joint without osteoarthritic changes. For correction of a severe hallux valgus we prefer modified Lapidus arthrodesis between the first MTT base and the medial cuneiform. In case of a reducible defect we perform a salvage operation on the first MTP joint, soft tissue techniques often in combination with the Akin osteotomy of the proximal phalanx. Arthrodesis is the method of choice in irreducible hallux valgus as well as in patients with osteoarthritis. In a marked varus deformity of the first MTT we prefer a twolevel arthrodesis. In combination with modified Lapidus arthrodesis we correct the axis of the whole first ray, excessive hallux valgus angle as well as 1-2 intermetatarsal angle. Our experience has shown that this procedure minimizes the risk of recurrence of the deformity.

A number of fixation devices can be used for arthrodesis of the first MTP joint and the joint between the first MTT and the medial cuneiform. The simplest of them include two or more Kirschner wires, cerclage wiring, fixation with one lag screw inserted in a retrograde or 
antegrade manner. The joint may be fixed with memory staples, two crossed screws and, last but not least, with numerous types of plates, often in combination with a lag screw placed plantarward. Based on multiple studies assessing the stability of individual systems, the most stable is considered to be fixation by LCP in combination with an interfragmentary screw.

In our study we performed arthrodesis of the first MTP joint by means of Variable Angle LCP 1st MTP Fusion Plate 2.4/2.7 (Synthes, Switzerland), which we have used since 2010 with excellent results ${ }^{8}$.

In addition to clinical studies, several other studies may be found in the literature, dealing with biomechanical tests on cadavers or synthetic bone. Synthetic bone used for biomechanical testing usually has constant mechanical properties. In cadaveric specimens various bone density has to be taken into account. When comparing 2 different methods, the right and the left cadaveric specimens should be used from the same donor in order to ensure equal conditions. The advantage of synthetic bone is its constant size; in cadavers length and width of specimens must be recalculated. Synthetic bone is easy to handle, without necessity of dissection of soft tissues. Several clinical studies have compared the properties of synthetic bone and cadaveric specimens and come to the conclusion that synthetic bones are an adequate alternative in biomechanical tests ${ }^{9-12}$.

In our study the cadaveric specimen was cleared of all soft tissues; joint capsule and collateral ligaments of the first MTP joint were removed, while articular cartilage was left in place. Because this was an experiment, we only wanted to test the stiffness of the fixation and not the stiffness of the joint capsule ligament. The outcome of the experiment would be affected by the different length, width and strength of individual cadaveric ligaments.

Foote et al. in their biomechanical study of the first MTP joint arthrodesis using Sawbones, compared 3 fixation constructs, including 2 crossed screws, a straight dorsal plate fixed by $2+2$ screws and a lag screw; and an $\mathrm{X}$ - Locking Plate with $2+2$ screws. They used 24 specimens that they treated with arthrodesis in 15 degrees of valgus and 20 degrees of dorsiflexion. On the Instra 5500 testing machine they used 1000 cycles to simulate gait with $100 \mathrm{~N}$ proximodistal force. The most stable construct was a straight dorsal plate with a lag screw followed by 2 crossed screws; arthrodesis with an X-Locking Plate showed the lowest stiffness ${ }^{13}$. Politi et al. also used synthetic bone in their study to compare 5 operative techniques to promote arthrodesis of the first MTP joint. The most stable technique was the combination of a four hole dorsal plate with an interfragmentary screw and conical reaming ${ }^{14}$.

In their study of 1993, Curtis et al. compared 4 methods of fixation for arthrodesis of the first MTP joint performed on 10 pairs of cadaveric feet, in 25 degrees of valgus and 15 degrees of dorsiflexion. In 3 fixation methods ( 2 Kirschner wires, dorsal plate placed longitudinally in combination with an interfragmentary screw, 1 screw), joint surfaces were resected perpendicular to the longitudinal axis. In the fourth technique they used powered coni- cal reamers for excision of the joint surfaces and fixation with one screw. They proved that bony preparation with power conical reamers significantly increased stability as compared to planar excision ${ }^{15}$. In their experimental study of 1986, Sykes et al. used 15 cadaveric pairs to compare 5 fixation techniques of arthrodesis of the first MTP joint, with the position of arthrodesis in 20 degrees of valgus and 20 degrees of dorsiflexion. The highest stability was achieved with fixation by 1 screw with planar excision of joint surfaces $(23.7 \mathrm{~N} / \mathrm{mm})$, and the least stable was fixation with a horizontal wire $(2.2 \mathrm{~N} / \mathrm{mm})\left(\right.$ ref. $\left.{ }^{16}\right)$. A similar cadaver study (12 pairs) was published by Buranosky et al. in 2001. In this study, all joint surfaces were prepared with power conical reamers. Arthrodesis with a dorsal 6-hole plate and an interfragmentary screw showed a significantly higher stability than the construct with 2 crossed screws ${ }^{17}$.

Different types of plates show different stability. Multiple differences were for instance revealed between a longitudinal plate and an X-Locking Plate. In a simple longitudinal plate, screws are aligned and placed at different intervals, and the plate mechanically reduces the load acting on the arthrodesis site. In an X-Locking Plate, the screws are placed at a constant distance close to the joint. In spite of this, an X-Locking Plate may be more beneficial for instance in short or osteoporotic bones. Fixation in metaphysis with residual cancellous bone is more stable. Sophisticated LCP constructs usually allow insertion of screws at a variable angle to a site with the highest bone quality. The use of LCP systems in osteoporotic patients is supported also by Kim et al. ${ }^{18}$.

Our method of choice of treatment of a severe irreducible hallux valgus deformity is a two-level arthrodesis. In order to minimize the risk of nonunion, Lapidus arthrodesis should be as stable as possible in these cases. Nonunion after Lapidus arthrodesis is reported at 5-15\% in the literature ${ }^{1}$. Particularly for this reason there appeared a number of experimental studies aimed at comparison of stability of the fixation.

In our biomechanical test on cadaveric specimens, we compared 2 methods of fixation of Lapidus arthrodesis, i.e. arthrodesis using 2 BOLD screws with arthrodesis by an X-Locking Plate fixed with $2+2$ screws. The result of the test has unequivocally proved that stiffness of the PS (plate-screw) fixation is considerably higher than that of the PP (plate-plate) construct, under all 3 loading modes.

Comparable results are reported by Cohen et al. who used 10 pairs of cadaver feet to compare fixation with a Normed $\mathrm{H}$ titanium locking plate and with 2 crossed 4.0 ACE DePuy cannulated screws. The mean stiffness of the construct with 2 crossed screws was significantly higher $(83.1 \mathrm{~N} / \mathrm{mm})$ as compared to that with the plate (19.96 $\mathrm{N} / \mathrm{mm}$ ) (ref. ${ }^{1}$ ). Gruber et al. in their study of 10 pairs of cadavers compared a four-hole LCP construct and a construct with one 4.0 cannulated screw and 2 crossed 4.0 cannulated screws. Biomechanical testing did not show any significant difference between the two methods ${ }^{19}$. In a cadaver study, Klos et al. used 8 pairs of cadaver feet to compare 2 methods of Lapidus arthrodesis, namely fixation with two $4.0 \mathrm{~mm}$ crossed screws versus fixation with 
a medial locking plate (X-Locking Plate 2.4/2.7, Synthes, Solothur, Switzerland) in combination with a $4.0 \mathrm{~mm}$ compression screw. As compared to other studies, they performed dynamic testing. The parameters measured included initial stiffness; plantar joint-line gapping after one cycle, 100 and 1000 cycles; and number of cycles to failure defined as more than or equal to $3 \mathrm{~mm}$ plantar gapping. According to the results the plate-and-screw construct showed significantly less plantar gapping and a higher number of cycles to failure as compared to the construct with 2 crossed screws ${ }^{5}$. Marks et al. compared fixation of Lapidus arthrodesis with 3 screws versus fixation using a four-hole plantar plate in combination with a Lisfranc screw (from the first metatarsal base to the second metatarsal base). The study documented a significantly higher stability of the screw-plantar plate construct (2917 N) as compared to the three-screw construct (1480 N). Plantar placement of the plate is technically more demanding but more beneficial in terms of biomechanics as it eliminates tensile forces on the plantar aspect ${ }^{20}$.

Placement of the plate dorsally is biomechanically least beneficial due to the forces acting primarily proximodistaly. Plantar placement of the plate eliminates plantar tensile forces, although such placement is unsuitable in terms of anatomical relations. A compromise may be medial or dorsomedial placement of the plate. It is useful to combine a dorsal plate with a plantar interfragmentary screw which significantly increases stability of arthrodesis.

Studies which included bone densitometry show similar results. The load to fixation failure is lower in specimens with a lower BMD. For instance, Klos found a statistically significant correlation between BMD and the number of cycles to failure ${ }^{5}$. Age-related decrease in stiffness of the specimen is given by degradation of bone material properties. In our experimental group we have proved a statistically significant age-related decrease in stiffness of the specimen $(P=0.004)$.

\section{CONCLUSION}

In case of a marked valgus deformity with osteoarthritis of the first MTP joint and varus deformity of the first MTT, we choose a two-level arthrodesis to correct the axis of the entire first ray of the foot, an excessive hallux valgus angle as well as the 1-2 intermetatarsal angle.

All cadaveric specimens were treated with arthrodesis of the first MTP joint in combination with arthrodesis of the first TMT joint according to modified Lapidus. Arthrodesis of the first MTP joint was performed with Variable Angle LCP $1^{\text {st }}$ MTP Fusion Plate 2.4/2.7. In case of PS (plate-screw) construct, we performed modified Lapidus arthrodesis with the use of 2 BOLD screws. In case of PP (plate-plate) construct, we implanted X-Locking Plate 2.4/2.7.

In all specimens the PS construct showed a statistically considerably higher stiffness than the PP construct. In all specimens treated with the PP construct the load to failure was lower than in the PS construct. For loading mode a., at a significance level of $0.05(P<0.05)$, the $\mathrm{p}$ - value was 0.036 , for mode $\mathrm{b}$. the $P$-value was 0.007 and for loading mode c. the $P$-value was 0.006 . In addition, agerelated decrease in stiffness of the specimen was proved at a significance level of $5 \%(P=0.004)$. The result confirmed our hypothesis.

Acknowledgement: The authors are pleased to thank Institute of Solid Mechanics, Mechatronics and Biomechanics and Institute of Anatomy of the Faculty of Medicine to manage this biomechanical study.

Author contributions: RK: came with the idea, study design, literature search, cadaver specimen prepare, wrote the manuscript; JK: study design, manuscript revision; TN,PV: cadaver specimen prepare, load testing process, data analysis; TP: manuscript revision; MJ, IC: help with cadaver specimens.

Conflict of interest statement: The authors state that they are no conflicts of interest regarding the publication of this article.

\section{REFERENCES}

1. Cohen DA, Parks BG, Schon LC. Screw fixation compared to H-locking plate fixation for first metatarsocuneiform arthrodesis: a biomechanical study. Foot Ankle Int. 2005;26(11):984-9.

2. Myerson M, Allon S, McGarvey W. Metatarsocuneiform arthrodesis for management of hallux valgus and metatarsus primus varus. Foot Ankle 1992;13(3):107-15.

3. Sangeorzan BJ, Hansen ST. Modified Lapidus procedure for hallux valgus. Foot Ankle 1989;9(6):262-6.

4. Roukis TS. Nonunion after arthrodesis of the first metatarsal-phalangeal joint: a systematic review. J Foot Ankle Surg Off Publ Am Coll Foot Ankle Surg 2011;50(6):710-3.

5. Klos K, Gueorguiev B, Mückley T, Fröber R, Hofmann GO, Schwieger K. Stability of medial locking plate and compression screw versus two crossed screws for lapidus arthrodesis. Foot Ankle Int 2010;31(2):158-63.

6. Popelka S, Vavrík P, Hromádka R, Sosna A. Lapidus procedure in patients with rheumatoid arthritis-short-term results. Z Orthop Unfall 2008;146(1):80-5. doi: 10.1055/s-2007-989439

7. Rippstein PF, Park YU, Naal FD. Combination of first metatarsophalangeal joint arthrodesis and proximal correction for severe hallux valgus deformity. Foot Ankle Int 2012;33(5):400-5. doi: 10.3113/ FAI.2012.0400

8. Kunovský R, Pink T, Jarošík J. Arthrodesis of the First Metatarsophalangeal Joint by Locking Plate. Acta Chir Orthop Traumatol Cech 2017;84(6):453-61.

9. Heiner AD. Structural properties of fourth-generation composite femurs and tibias. J Biomech 2008;41(15):3282-4.

10. Heiner AD, Brown TD. Structural properties of a new design of composite replicate femurs and tibias. J Biomech 2001;34(6):773-81.

11. Cristofolini L, Viceconti M. Mechanical validation of whole bone composite tibia models. J Biomech 2000;33(3):279-88.

12. Dunlap JT, Chong ACM, Lucas GL, Cooke FW. Structural properties of a novel design of composite analogue humeri models. Ann Biomed Eng 2008;36(11):1922-6.

13. Foote KM, Teasdall RD, Tanaka ML, Scott AT. First metatarsophalangeal arthrodesis: a biomechanical comparison of three fixation constructs. J Surg Orthop Adv 2012;(4):223-31.

14. Politi J, John H, Njus G, Bennett GL, Kay DB. First metatarsal-phalangeal joint arthrodesis: a biomechanical assessment of stability. Foot Ankle Int 2003;24(4):332-7.

15. Curtis MJ, Myerson M, Jinnah RH, Cox QG, Alexander I. Arthrodesis of the first metatarsophalangeal joint: a biomechanical study of internal fixation techniques. Foot Ankle 1993;14(7):395-9.

16. Sykes A, Hughes AW. A biomechanical study using cadaveric toes to test the stability of fixation techniques employed in arthrodesis of the first metatarsophalangeal joint. Foot Ankle 1986;7(1):18-25. 
17. Buranosky DJ, Taylor DT, Sage RA, Sartori M, Patwardhan A, Phelan M First metatarsophalangeal joint arthrodesis: quantitative mechanical testing of six-hole dorsal plate versus crossed screw fixation in cadaveric specimens. J Foot Ankle Surg Off Publ Am Coll Foot Ankle Surg 2001;40(4):208-13.

18. Kim T, Ayturk UM, Haskell A, Miclau T, Puttlitz CM. Fixation of osteoporotic distal fibula fractures: A biomechanical comparison of locking versus conventional plates. J Foot Ankle Surg Off Publ Am Coll Foot Ankle Surg 2007;46(1):2-6.
19. Gruber F, Sinkov VS, Bae S-Y, Parks BG, Schon LC. Crossed Screws versus Dorsomedial Locking Plate with Compression Screw for First Metatarsocuneiform Arthrodesis: A Cadaver Study. Foot Ankle Int 2008;29(9):927-30.

20. Marks RM, Parks BG, Schon LC. Midfoot fusion technique for neuroarthropathic feet: biomechanical analysis and rationale. Foot Ankle Int 1998;19(8):507-10. 